\title{
Ultrasonography findings of vulvar liposarcoma. Case report.
}

\author{
Bahar Yanik $^{1}$, Umit Inceboz ${ }^{2}$, Erdogan Bulbul ${ }^{1}$, Gulen Demirpolat ${ }^{1}$, Ismail Engin Uzgoren ${ }^{3}$
}

${ }^{1}$ Department of Radiology, Balikesir University Faculty of Medicine, ${ }^{2}$ Department of Obstetrics\&Gynecology, Balikesir University Faculty of Medicine, ${ }^{3}$ Balikesir Uzgoren Pathology Laboratory, Balikesir, Turkey

\begin{abstract}
Liposarcoma (LPS) of vulva is a rare entity. We present the ultrasonographic (US) and color Doppler ultrasonographic (CDUS) findings of a vulvar myxoid LPS. Although LPS cases have been reported in the extremities and trunk, the US or CDUS findings of LPS in vulva have not been described previously. On US the mass appeared as a well-defined, homogeneous hypoechoic structure and on CDUS it was quite hypervascular.

Keywords: liposarcoma, vulva, ultrasonography, color Doppler
\end{abstract}

\section{Introduction}

Among vulvar malignancies, liposarcomas (LPS) are extremely rare; in fact most of the vulvar neoplasms are squamous cell carcinomas [1,2]. Vulvar LPS is difficult to be accurately diagnosed preoperatively and may be misinterpreted as benign lesions due to their close resemblance to Bartholin's gland pathologies such as cysts or abscesses, inguinal or femoral hernias, or benign softtissue tumors as a result of their location and appearance. Imaging modalities such as ultrasonography (US), color Doppler ultrasonography (CDUS), computed tomography (CT) and magnetic resonance imaging (MRI) have been used to assess soft tissue tumors $[3,4]$. Especially US is the first line examination technique in vulvar pathologies. US findings of LPS may be nonspecific in other parts of body, because lesions present with variable, nonspecific patterns. Clinical presentation and pathological findings have been demonstrated previously; however, to the best of our knowledge, US and CDUS findings

Received 23.10.2014 Accepted 10.11.2014

Med Ultrason

2015, Vol. 17, No 3, 398-400

Corresponding author: Erdoğan Bülbül, MD

Balıkesir University, Medicine Faculty,

Department of Radiology

Cagis Yerleskesi, Bigadic yolu 17.km 10145

Altieylul, Balıkesir, Turkey

Phone: 090.505 2204560, Fax: 090.266.6121459

E-mail: drerdoganbulbul@yahoo.com of a vulvar LPS have not been described in the literature before. In this case report, US and CDUS findings of a patient with a vulvar myxoid LPS are presented.

\section{Case report}

A 52-year old female was admitted with a complaint of a rapidly growing painful mass in the vulva. She had noticed this mass two months ago. In the past, she had not received irradiation to the vulva. There was no history of cancer in her family. At gynecological examination vagina, cervix and uterus appeared normal. A vulvar lump was observed (maximum diameter $5 \mathrm{~cm}$ ). The overlying skin of the mass looked normal. At palpation, this mass was firm and sharply circumscribed in the posterior part of the left labium majus. No inguinal lymphadenopathy was noted. The gray-scale US and CDUS of the vulva was performed with a SSA-780A ultrasound scanner (Toshiba, Ottawa, Japan) and a 6-11 MHz multifrequency linear-array transducer. A prominent homogenous hypoechoic and ovoid solid mass with well-defined borders of $52 \times 25 \mathrm{~mm}$ diameter was detected in the subcutaneous fat tissue in the left labium majus. Mild marginal scalloping was observed posteriorly and posterior enhancement was detected (fig 1a). CDUS and power Doppler US showed arterial blood flow with low resistance blood flow and high systolic peaks $(70 \mathrm{~cm} / \mathrm{s})$ in this mass (fig $1 \mathrm{~b}, \mathrm{c})$. The patient refused to undergo magnetic resonance imaging (MRI). The mass was removed by excisional biopsy. At 


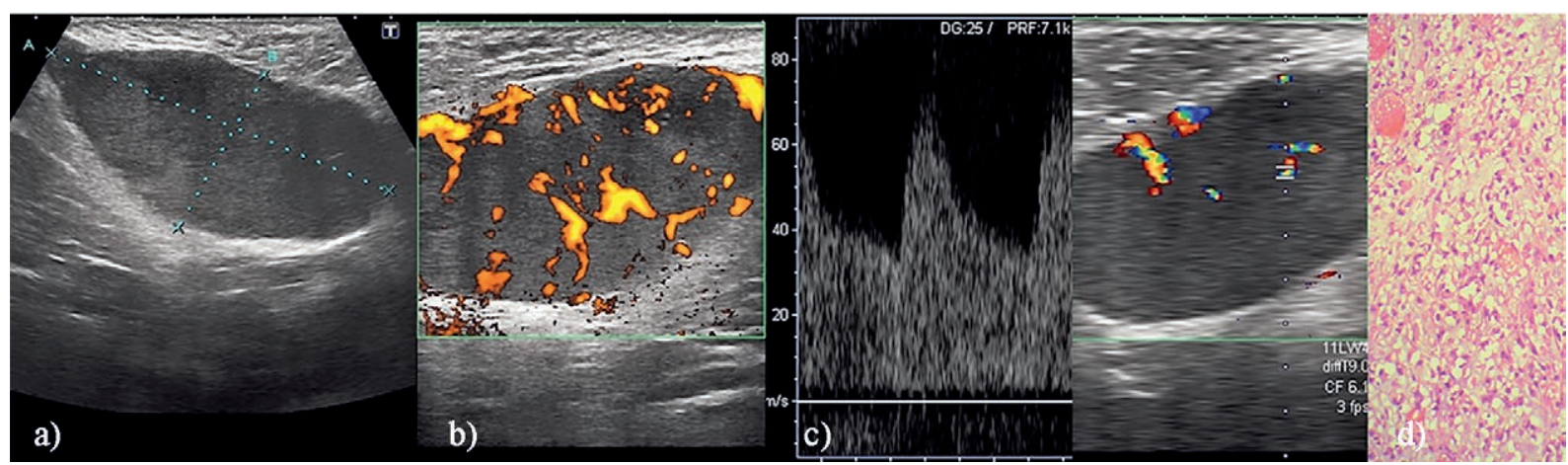

Fig 1. a) gray scale US examination revealed a prominent homogenous hypoechoic and ovoid solid mass with well-defined borders; b) power Doppler US showed central and peripheral vascularization in the mass; c) in spectral analysis, high peak systolic velocity $(70 \mathrm{~cm} / \mathrm{s})$ and low resistance blood flow pattern were observed; d) on histopathological examination, the tumor consisted of small cells within a myxoid matrix with anastomosing small vessels and rare adipocytes (H\&E 10x20).

operation the tumor was not attached to the overlying skin but posterior margin adhered to underlying muscle fascial planes, consequently causing difficulty in surgical resection. Histopathological analysis, revealed a myxoid LPS (fig 1d). The patient was discharged without complications after five days. Computed tomography (CT) was performed for staging and no regional or distant metastasis was observed. Two years after operation the patient had no signs of recurrence.

\section{Discussions}

Primary LPS of the vulva is extremely rare, representing $<1 \%$ of all LPSs [5]. The etiology of soft tissue sarcomas has not yet been clearly defined [6]. According to the recent developments in molecular biology and genetic mapping, LPS can be divided into three categories: atypical lipomatous tumor, well-differentiated LPS, and dedifferentiated LPS [3]. The second category, which occurs in young adults, includes myxoid LPS and round cell LPS. The third category is composed of pleomorphic LPS which is the least common subtype of LPS [3].

Myxoid LPS often shows some degree of nuclear atypia and hyperchromasia of the neoplastic cells. Lipoblastomas share some histopathological characteristics with myxoid LPS [7]. Myxoid LPS should be distinguished from lipoblastoma which is a benign mesenchymal tumor composed of various stages of maturing adipocytes. In myxoid LPS, the maturing adipocytes are located at the periphery of lobules instead of the center as seen in lipoblastoma [8]. Another diagnostic clue may be the patient's age. Although lipoblastoma is typically encountered in infants less than 3 years of age, it has been reported in adults too $[9,10]$.

Several radiological modalities have been used to determine a soft tissue tumor, such as US, CDUS, CT,
MRI, and angiography. US and CDUS have gained acceptance in the evaluation of soft tissue tumors within the last thirty years [11] being a noninvasive, available, and inexpensive technique relative to other imaging modalities. Owing to the use of high-resolution and wide range frequency transducers and advances in CDUS, the combination of US and CDUS examination is still the first imaging modality for the detection of soft tissue tumors from the skin to the surface of the bones. Moreover, USguided needle biopsy together with clinical and US examination can provide a cost-effective shortcut to a final diagnosis $[11,12]$. There are other advantages of US and CDUS over MRI. Firstly dynamic US examination can be performed using patient movement, lesion compression, or muscle contraction. Secondly subtle calcification in the tumors can be shown easily and lesional vascularization can be demonstrated without using a contrast agent. One advantage over the $\mathrm{CT}$ is the lack of radiation exposure involved. However, a multimodality approach is often needed for further characterization and staging of soft tissue tumors $[12,13]$. A MRI examination could not be performed preoperatively in our patient.

Soft tissue sarcomas are predominantly hypoechoic at US. The exception of this condition is some LPS $[12,14]$. The echogenicity of LPS differs depending on the cell type $[4,14]$. Myxoid areas are hypoechoic where retained lipoblastic nets are hyperechoic at US [10,14]. Therefore, myxoid LPS are often heterogeneous and hypoechoic. Sometimes it is difficult to distinguish radiologically the myxoid LPS from lipoblastoma. The imaging appearance of lipoblastoma reflects the underlying pathologic features and varies depending on the extent of fat versus myxoid stroma. Especially, the myxoid components may predominate with only small elements of fat in infants. Lipoblastomas with this composition are indistinguishable from a myxoid LPS at US. In a recent study Chiou 
et al used high-resolution US and CDUS to evaluate benign and malignant soft-tissue tumors [14]. According to this study, typical US pattern of LPS was heterogeneous hyperechogenicity, infiltrated margins, scalloped shape, and solid composition. In our case, the mass was homogeneously hypoechoic at US. This appearance might be caused by the high myxoid component of the tumor.

CDUS may add specificity in US evaluation of soft tissue tumors. Increased, chaotic vascularity is observed in soft-tissue sarcomas on CDUS. But some tumors may remain totally avascular, even when examined with the most sensitive power Doppler US [12]. Rich capillary network was described in the myxoid areas histologically [10]. Myxoid LPS may be observed as a vascular lesion on CDUS due to this rich capillary network. Chiou et al reported that moderate/high vascularity in the masses were detected on CDUS in 14 patients with LPS [4]. They did not find avascular pattern in any of the patients with LPS. Additionally Belli et al pointed out a correct diagnosis can be obtained with a $90 \%$ sensitivity and $91 \%$ specificity when a threshold of $50 \mathrm{~cm} / \mathrm{s}$ was identified for systolic velocity to differentiate benign tumors from malignants [15]. In our case, the mass was hypervascular and demonstrated high peak systolic velocity $(70 \mathrm{~cm} / \mathrm{s})$, high end diastolic velocity, and low resistance blood flow. A regular arrangement of vessels with a linear course is suggestive of a benign mass. In contrast, randomly distributed vessels with abrupt variations in size as well as spot flow signals are fairly typical of malignancy [15]. In our case multiple irregularly arranged and spot flow signals were observed.

In conclusion, US and CDUS findings of vulvar LPS resemble the ones in the other parts of the body. These findings are those of a mass with a nonspecific solid appearance, whose nature cannot be recognized. Combination of other radiological modalities, may aid in the characterization of the mass. However, generally definitive diagnosis ultimately requires histopathological examination.

\section{References}

1. Rogers RG, Thorp JM Jr. Liposarcoma of the vulva. J Reprod Med 1995; 40: 863-864.
2. Deppe G, Mert I, Winer IS. Management of squamous cell vulvar cancer: a review. J Obstet Gynaecol Res 2014; 40: 1217-1225.

3. O'Regan KN, Jagannathan J, Krajewski K, et al. Imaging of liposarcoma: classification, patterns of tumor recurrence, and response to treatment. AJR Am J Roentgenol 2011; 197: W37-W43.

4. Chiou HJ, Chou YH, Chiu SY, et al. Differentiation of benign and malignant superficial soft-tissue masses using gray scale and color Doppler ultrasonography. J Chin Med Assoc 2009; 72: 307-315.

5. Nucci MR, Fletcher CD. Liposarcoma (atypical lipomatous tumors) of the vulva: a clinicopathologic study of six cases. Int J Gynecol Pathol 1998; 17: 17-23.

6. Loubignac F, Bourtoul C, Chapel F. Myxoid liposarcoma: a rare soft tissue tumor with a misleading benign appearance. World J Surg Oncol 2009; 7: 42.

7. Lae ME, Pereira PF, Keeney GL, Nascimento AG. Lipoblastoma-like tumor of vulva: report of three cases a distinctive mesenchymal neoplasm of adipoctic differentiation. Histopathology 2002; 40: 505-509.

8. Kirkham YA, Yarbrough CM, Pippi Salle JL, Allen LM. A rare case of inguinolabial lipoblastoma in a 13-month-old female. J Ped Urol 2013; 9: e64-e67.

9. Kransdorf MJ, Moser RP Jr, Meis JM, Meyer CA. Fat-containing soft-tissue masses of the extremities. Radiographics 1991; 11: 81-106.

10. Murphey MD, Carroll JF, Flemming DJ, Pope TL, Gannon FH, Kransdorf MJ. From the archives of the AFIP: benign musculoskeletal lipomatous lesions. Radiographics 2004; 24: 1433-1466.

11. Choi H, Varma DG, Fornage BD, Kim EE, Johnston DA. Soft-tissue sarcoma: MR Imaging vs sonography for detection of local recurrence after surgery. AJR Am J Roentgenol 1991; 157: 353-358.

12. Fornage BD. Soft-tissue masses: the case for increased utilization of sonography. Appl Radiol 2000; 29: 8-22.

13. Pike J, Clarkson PW, Masri BA. Soft tissue sarcomas of the extremities: how to stay out of trouble. BCMJ 2008; 50: 310-318.

14. Fornage BD. Soft tissue masses: the underutilization of sonography. Semin Musculoskelet Radiol 1999; 3: 115-134.

15. Belli P, Costantini M, Mirk P, Maresca G, Priolo F, Marano P. Role of color Doppler sonography in the assesment of musculoskeletal soft tissue masses. J Ultrasound Med 2000; 19: 823-830. 